\title{
ALL-SOLID-STATE 3-D RECHARGEABLE LITHIUM BATTERIES WITH SILICON ROD STRUCTURED ELECTRODE
}

\author{
Jian Wang , James E. Trevey, Se-Hee Lee, and Victor M. Bright* \\ University of Colorado, Boulder, Colorado, USA
}

\begin{abstract}
This paper presents 3-D MEMS-fabricated lithium rechargeable batteries relying on structured silicon rods as anodes in order to increase the effective electrode area. A new method of fabricating silicon micro/nano rod arrays with controllable diameters in the range of $300 \mathrm{~nm}-8 \mu \mathrm{m}$ is proposed. $2 \mu \mathrm{m}$ silicon rods as anodes were successfully incorporated into an all-solidstate battery construction. The structured architecture of the silicon electrodes allowed for a heightened surface area as well as improvement in cycle life and capacity over comparable planar electrodes. Structured rod array electrodes showed a first cycle columbic efficiency over $80 \%$ which is more than double that of conventional powder composite silicon electrodes. Galvanostatic cycling of structured silicon rod electrodes showed highly reversible capacity for high current densities.
\end{abstract}

\section{INTRODUCTION}

Mounting electric energy sources on semiconductor chips is highly attractive due to rapid growth of electrical and mechanical integration technologies such as wireless telecommunications and emerging integrated optoelectronic circuits. Planar solid-state thin film batteries are widely used as an energy source in current MEMS and CMOS devices but are incapable of meeting growing power demands [1]. Recent technological advancements have realized improved battery performance by reconfiguring electrode materials from the typical 1-D battery design of commercial technology into 3-D architectures [2]. Such battery configurations more effectively utilize the highly limited space on board of MEMS and CMOS devices by increasing the power capabilities of batteries without increasing their footprint.

The binary silicon-lithium material system shows the highest specific capacities up to $4,200 \mathrm{mAh} \mathrm{g}^{-1}$ (which corresponds to 4.4 mol of $\mathrm{Li}$ per mol of $\mathrm{Si}$ ) [3]. Unfortunately, the reversibility of conventional Si material at room temperature is poor, due to the expansion and contraction of lithium silicide during the chargedischarge process that results in the pulverization of the Si material [4-6]. As a consequence, the electrical contact between the current collector and the Si material fails, leading to a rapid decrease in the system capacity after several cycles. Many reports have focused on reducing such volume change via conductive additives that act as an electron conductor between pulverized particles. This approach however, requires that a high percent of the electrode be a noncontributing material, thereby largely reducing the maximum attainable capacity of the battery [7]. In principle, a reduction in the size of the Si material (i.e. from micro- to nano-sized crystals) should mitigate the reversibility problem, since a smaller $\mathrm{Si}$ crystallite will have greater surface to volume ratio and more surface area in contact the current collector, even after lattice expansion and contraction during cycling. To this end, the binary $\mathrm{Li}-\mathrm{Si}$ system has been previously investigated to a limited extent, with initial work demonstrating superior discharge capacity for micro- and nano-sized Si materials [8]. In general, this approach offers initial evidence for the improved performance of small-scale $\mathrm{Si}$ materials for the Li-Si anode system over conventional battery materials [4]. Here, we show that silicon rod structured battery electrodes circumvent the pulverization issues as they can accommodate large strain with minimal pulverization, providing good cycle life and relatively high columbic efficiency.

\section{DESIGN AND ROD ARRAY FABRICATION}

In this work we investigate the fabrication and use of 3-D integrated all-solid-state lithium batteries with structured $\mathrm{Si}$ rod electrodes and powder solid-state electrolyte (figure 1). Using a ntype (Sb doped) single side polished, 3 inch diameter silicon wafer (<100> cut, $350 \mu \mathrm{m}$ thick, 0.008 0.02 $\Omega \mathrm{cm}$ ), Si micro-rods are

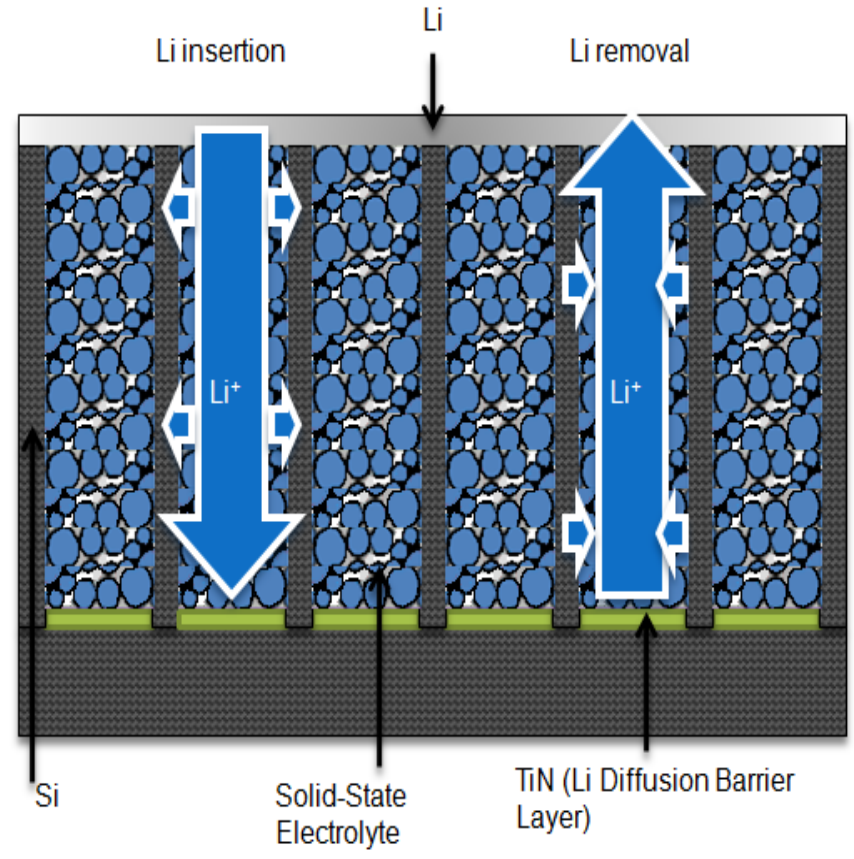

Figure 1: Cross-section view of our 3D integrated all-solid-state battery.

obtained by deep reactive ion etching (DRIE: STS Pegasus DRIE system), and made thinner by subsequent RIE (Plasmatherm 540/540 Dual Chamber RIE system). The fabrication process of the rod array is shown in figure 2. After spin coating and photo lithography, the photoresist (AZ 4210) is hard baked in a vacuum oven for 10 minutes before using DRIE to obtain the Si rod array. The diameter of the rods can be controlled to within the range of 3 $\mu \mathrm{m}-8 \mu \mathrm{m}$ by using different masks. Following DRIE, rods are made thinner by subsequent $\mathrm{SF}_{6} \mathrm{RIE}$ at an etching rate of 400-600 $\mathrm{nm} / \mathrm{min}$. At this step, $400 \mathrm{~nm}$ copper is thermally evaporated (3_LTEC MED 020 Coating System) onto the sample as a metallic mask layer for the RIE process. After RIE, the copper layer is removed by copper etchant and then a $70 \mathrm{~nm}$ layer of $\mathrm{Ti}$ is evaporated on top of the Si substrate and the rods. Ti is turned into a TiN barrier layer by heating the sample in $\mathrm{N}_{2}$ for 1 minute at 800 ${ }^{\circ} \mathrm{C}$. Due to the high aspect ratio of the gap, the TiN barrier will only cover the substrate and the top of the rods but not the side wall as proved by figure 3 . A TiN Li diffusion barrier layer is formed on the $\mathrm{Si}$ substrate for the purpose of preventing the lithiation of Si substrate, in order to avoid mechanical failure of the bulk Si substrate during the cycling [9]. After the TiN barrier layer is coated, $\mathrm{SF}_{6} \mathrm{RIE}$ is used again to obtain the desired dimension of 
(a)

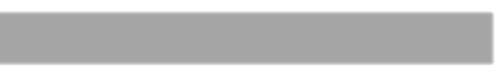

(b)

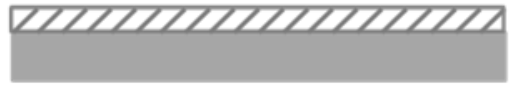

(c)

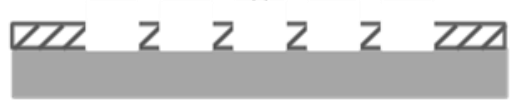

(d)

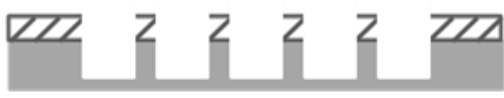

(e)

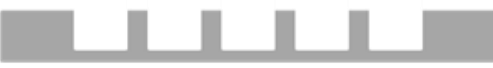

(f)

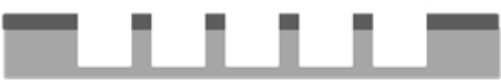

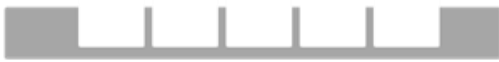

(h)

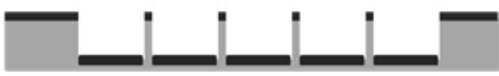

(i)

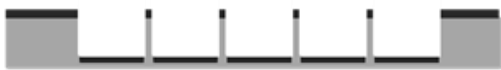

Figure 2. Fabrication process for creating Si rod array.(a) Silicon wafer (n-type, Sb-doped, 100 cut, 350 um thick 0.008 0.02 ohm-cm)(b) Spin coating 2.5 um Photoresist (AZ 4210) on Silicon wafer (c) Photo lithography and PR hard baking (d) DRIE for 8 mins (etching rate $3 \mu \mathrm{m} / \mathrm{min})($ (e) Remove PR and clean the sample (Acetone, API clean first, then Plasma clean) (f) Thermal evaporate metallic mask(Copper) (g) $\mathrm{SF}_{6}$ RIE and remove Copper ( $h$ ) Thermal evaporate Ti and heat the sample in $N_{2}$ atmosphere (i) $\mathrm{SF}_{6} \mathrm{RIE}$

the rods. The diameter of the rods is controlled by the etching time of RIE. Figure 4 shows the final result of a fabricated rod array. From (a) to (d), the diameters of the rods are respectively $300 \mathrm{~nm}$, $750 \mathrm{~nm}, 2000 \mathrm{~nm}$ and $8000 \mathrm{~nm}$. The gap between the rods can also be controlled by using different mask in the lithography process. Here, we choose the rod array with the diameter of $2 \mu \mathrm{m}$, height of $20 \mu \mathrm{m}$ and gap of $10 \mu \mathrm{m}$ for battery demonstration.

Si

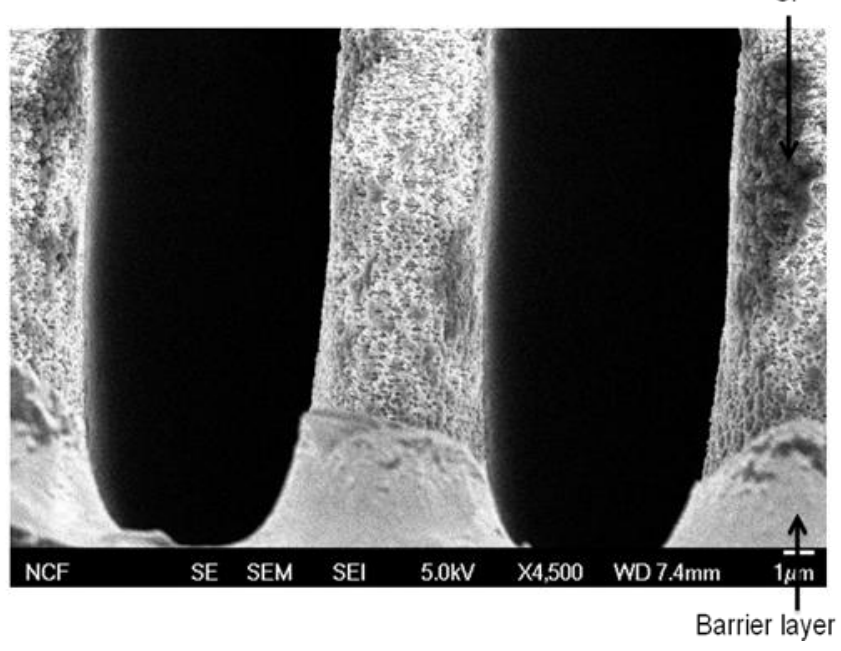

Figure 3. SEM image of Si rods with TiN barrier layer coated on the substrate but not the side wall of the rod

\section{MATERIALS AND CHEMISTRY}

\section{Solid Electrolyte}

Reagent-grade powders of $\mathrm{Li}_{2} \mathrm{~S}$ (Aldrich, 99.999\%) and $\mathrm{P}_{2} \mathrm{~S}_{5}$ (Aldrich, 99\%) were used as starting materials for mechanical ball milling. We produce $77.5 \mathrm{Li}_{2} \mathrm{~S}-22.5 \mathrm{P}_{2} \mathrm{~S}_{5}(\mathrm{~mol} \%)$ by the single step ball milling (SSBM) process [10]. Appropriate concentrations of materials are deposited into a zirconia vial (Spex) at a net weight of one gram with 2 zirconia balls (1x 12mm, 1x15mm in diameter) for grinding. High energy ball milling (Spex2000) took place for 20 continuous hours in an $\mathrm{Ar}$ filled glove box at $55{ }^{\circ} \mathrm{C}$. The resulting powder is recovered and used without further alterations for solid state batteries.

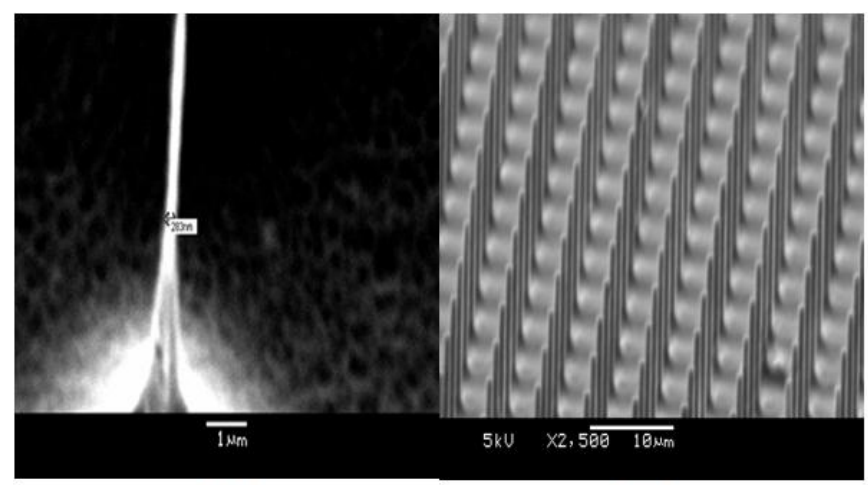

(a)

(b)

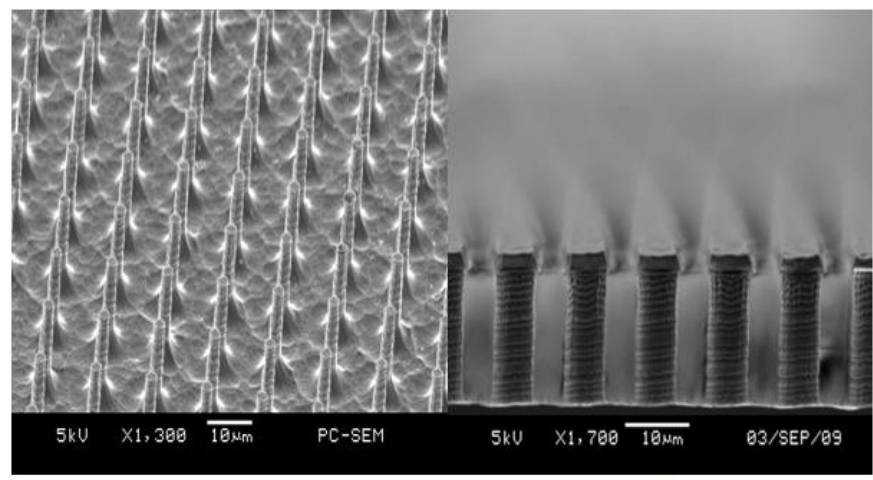

(c)

(d)

Figure 4. SEM image of Si rods with the diameter in the range of $300 \mathrm{~nm} \sim 8 \mathrm{~m}$ on Si substrate. The diameters of the rod from (a) to (d) are respectively $300 \mathrm{~nm}, 750 \mathrm{~nm}, 2000 \mathrm{~nm}$ and $8000 \mathrm{~nm}$.

\section{Battery Chemistry}

Lithium batteries are developed and tested in this study. The rechargeable batteries are simple in design, as well as safe and robust. Its all-solid-state construction prevents lithium dendrites from growing through the electrolyte, as happens with liquid-based electrolyte systems, thereby enhancing the operation safety. The reactions for the electrodes during operation include:

$$
\begin{gathered}
L i \Leftrightarrow L i^{+}+e^{-} \\
S i+L i^{+}+e^{-} \Leftrightarrow L i S i
\end{gathered}
$$




\section{BATTERY ASSEMBLY AND TESTING Assembly}

A cross sectional schematic of the battery assembly process composed of two electrodes separated by an electrolyte cavity can be seen in Fig. 5. In the lithium battery, the bottom electrode is comprised of just the silicon wafer and rod array while the top electrode is simply lithium metal, both in contact with titanium current collectors.

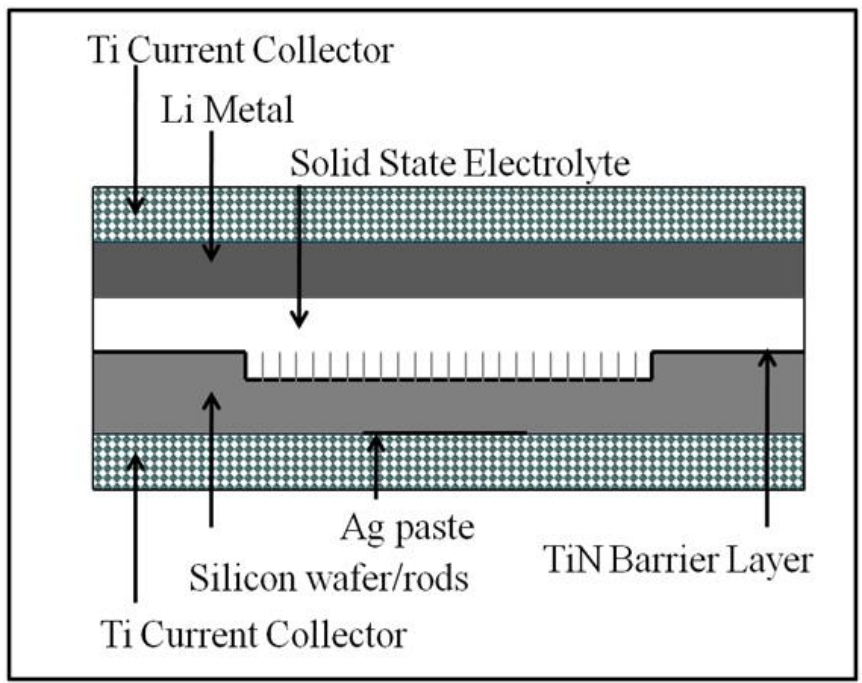

Figure 5: Schematic of battery construction. The lithium battery employing the silicon wafer/rods in a cathode design

The lithium batteries are formed by first attaching the silicon substrate to a titanium current collector with a conductive silver paste to ensure good contact. After the conductive paste has cured, solid electrolyte is weighed and poured into the die. The silicon wafer/solid electrolyte are pressed together in the die at 5 metric tons for approximately 5 minutes. A lithium foil is then attached to the solid state electrolyte at a pressure of 1 metric ton.

\section{Testing}

Batteries were characterized by using galvanostatic cycling to observe their charge-discharge behavior. Batteries were cycled at varying current densities to observe performance and degradation at high rates. Most commonly, batteries are charged and discharged between preset voltage limits, called constant voltage cycling. We employed constant capacity cycling limits so that structured silicon rods were lithiated to a capacity correlating to approximately $3600 \mathrm{mAh} \mathrm{g}^{-1}$, which is the room temperature capacity limit for silicon as an electrode material in all solid state batteries. In general, constant capacity cycling is used to determine materials' potential limits for the first few cycles and will be followed by constant voltage cycling to observe degradation. Our experiments, using continued constant capacity cycling perform the same task but allow for closer observation of structural transformations of materials.

\section{RESULTS}

\section{Columbic efficiency}

Figures 6 shows the electrochemical data for our 3D integrated all-solid-state lithium battery for the first 20 cycles and data for a comparable state-of-the-art 1D battery for comparison [10]. The columbic efficiencies of the first cycle of structured 3D batteries are $70 \%$ and $82 \%$ at an applied current of $1250 \mu \mathrm{Ah} / \mathrm{cm}^{2}$ and 250 $\mu \mathrm{Ah} / \mathrm{cm}^{2}$ respectively (Fig. 6A). The high value of the columbic efficiency of the first cycle is most fascinating because the generally accepted value for the columbic efficiency of batteries with Si based electrodes is closer to $35 \%$ due to the excessive pulverization of electrode material. Columbic efficiency is a reading of how well a battery is transporting lithium. $100 \%$ columbic efficiency implies that all of the lithium that was transported from the lithium metal to the silicon electrode is transported back to the lithium metal. In the case of low columbic efficiency, some lithium is being trapped in the electrode. Continued cycling revealed a relatively stable columbic efficiency that indicates lithium trapping and degradation in the $\mathrm{Li}_{\mathrm{x}} \mathrm{Si}$ electrode is minimal during cycling.

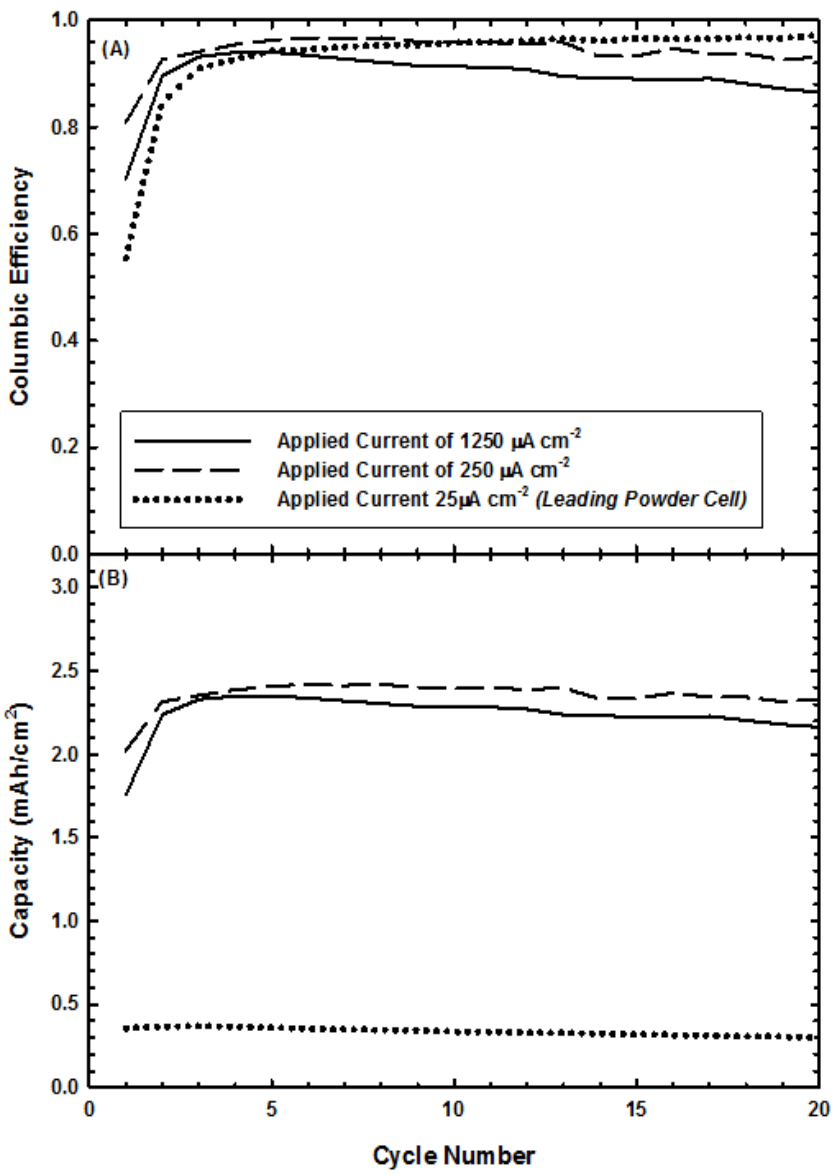

Figure 6. (A) Electrochemical data for our 3D integrated all-solidstate battery and comparison with state-of-the-art battery, columbic efficiency versus cycle number at different applied currents. (B) Electrochemical data for our 3D integrated all-solidstate battery and comparison state-of-the-art battery, capacity versus cycle number at different applied currents.

\section{Capacity}

Figure 6B shows again a comparison of our all-solid-state lithium batteries verses a current state-of-the-art battery, except for capacity at various applied currents instead of columbic efficiency. An approximate 8 fold improvement in capacity is observed for our rod structured battery over the leading technology. We have shown significant improvement of not only the overall capacity of potential on-board batteries but we have achieved high columbic efficiency on the first cycle of a Si based electrode. 


\section{Cycle life}

$\mathrm{Si}$ Micro/Nano rod structures were found to exhibit a longer cycle life than bulk Si [4]. Our rod-structured battery displays constant capacity with minimal degradation for over 20 cycles. As lithium trapping and pulverization isolate $\mathrm{Si}$ upon cycling, lithium diffuses deeper into the rods for conducting Si with which to react. As more $\mathrm{Si}$ becomes isolated, a larger resistance is observed. Figure 7 shows this trend of increasing resistance with a decreasing low cutoff voltage of each cycle. The structured nature of the rod array electrodes allows for minimal resistance layer build-up, as the rods can accommodate large strain with minimal pulverization.

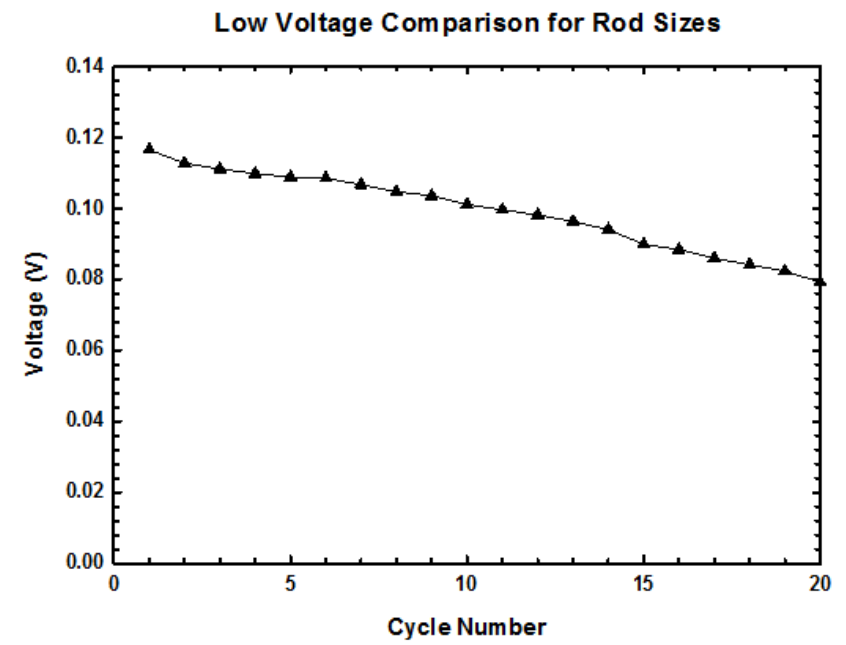

Figure 7. The low cut-off voltage of each discharge cycle.

\section{DISCUSSION}

Rechargeable lithium batteries were produced and tested in this work with a fair degree of repeatability. Inconsistencies arose with imperfections in our method manufacturing rod arrays as well as battery assembly. Rod array dimensions were difficult to fabricate precisely with accuracy within $100 \mathrm{~nm}$ of the target dimension, solid electrolyte filling of rod cavity for perfect contact was not verified, and TiN coating effectiveness were all areas in need of further investigation. Future work will focus on repeatability of size dimensioning of rod arrays and of battery assembly, as well as TiN coating effectiveness.

\section{CONCLUSION}

In this work, 3-D MEMS-fabricated lithium batteries incorporating structured silicon rods are designed, fabricated and tested. A simple, quick, and repeatable micro/nano rod array fabrication process for the battery anodes is developed by our group using traditional MEMS technology. The testing results of the 3-D lithium battery showed a fair degree of repeatability and revealed that structured silicon rod arrays achieve a high first cycle as well as high continued cycling columbic efficiency. High capacity with high reversibility was observed for lithium batteries. Long cycle life with little fading was achieved by using rodstructured electrodes.

\section{ACKNOWLEDGEMENTS}

This work was supported by the Semiconductor Research Corporation (SRC). Special thanks to Dr. Ann Li at NIST for assistance with DRIE. Partial support by DARPA Center on Nanoscale Science and Technology for Integrated Micro/NanoElectromechanical Transducers (iMINT) funded by DARPA
N/MEMS S\&T Fundamentals Program (HR0011-06-1-0048)(Dr. D. L. Polla, Program Manager) is also acknowledged.

\section{REFERENCES}

[1] L. Baggetto, R. A. H. Niessen, F. Roozeboom, and P. H. L. Notten, "High Energy Density All-Solid-State Batteries: A Challenging Concept Towards 3D Integration", Adv. Funct. Mater, 18, 1057 (2008).

[2] J. W. Long, B. Dunn, D. R. Rolison, and H. S. White, "ThreeDimensional Battery Architectures". Chem. Rev., 104, 4463 (2004).

[3] M. Green, E. Fielder, B. Scrosati, M. Wachtler, J. S. Moreno, "Structured Silicon Anodes for Lithium Battery Applications", Electrochemical and Solid-State Letters, 6 A75 (2003).

[4] C. K. Chan, H. Peng, G. Liu, K. Mcllwrath, X. F. Zhang, R. A. Huggins, and Y. Cui, "High-performance Lithium Battery Anodes Using Silicon Nanowires", Nature Nanotechnology, 3, 31 (2008).

[5] M. H. Park, M. G. Kim, J. Joo, K. Kim, J. Kim, S. Ahn, Y. Cui, and J. Cho, "Silicon Nanotube Battery Anodes", Nano. Letter, 93844 (2009).

[6] K. Peng, J. Jie, W. Zhang, and S. T. Lee "Silicon Nanowires for Rechargeable Lithium-ion Battery Anodes", App. Phys. Lett., 93, 033105 (2008).

[7] H. Kim and J. Cho "Superior Lithium Electroactive Mesoporous Si@Carbon Core-Shell Nanowires for Lithium Battery Anode Material ", 8, 3688 (2008).

[8] H. Kim, B. Han, J. Choo, and J. Cho "Three-Dimensional Porous Silicon Particles for Use in High-Performance Lithium Secondary Batteries", 47, 10151 (2008).

[9] US patent, \# 5,236,868, Date Aug. 17, 1993

[10] J. E. Trevey, Y. S. Jung, S. H. Lee, “Glass-ceramic $\mathrm{Li}_{2} \mathrm{~S}_{-} \mathrm{P}_{2} \mathrm{~S}_{5}$ Electrolytes Prepared by a Single Step Ball Milling Process and Their Application for All-solid-state Lithium-ion Batteries", Electrochem. Comm., 11, 1830 (2009).

\section{CONTACT}

*Victor M. Bright, Victor.Bright@Colorado.EDU Tel: (303) 7351734 